\title{
MBE Growth of Al/InAs and Nb/InAs Superconducting Hybrid Nanowire Structures
}

\author{
Nicholas A. Güsken ${ }^{1,2,3}$, Torsten Rieger ${ }^{1,2}$, Benjamin Bennemann ${ }^{1}$, Elmar \\ Neumann $^{4}$, Mihail Ion Lepsa ${ }^{1,2}$, Thomas Schäpers ${ }^{1,2}$, Detlev Grützmacher ${ }^{1,2}$ \\ ${ }^{1}$ Peter Grünberg Institute (PGI-9), Forschungszentrum Jülich, 52425 Jülich, Germany \\ ${ }^{2}$ JARA-Fundamentals of Future Information Technology (JARA-FIT), Jülich-Aachen Research Alliance, Germany \\ ${ }^{3}$ Present address: Department of Physics, Imperial College London, London, SW7 2AZ, U.K. \\ ${ }^{4}$ Peter Grünberg Institute (PGI-8), Forschungszentrum Jülich, 52425 Jülich, Germany
}

\begin{abstract}
We report on in situ growth of crystalline $\mathrm{Al}$ and $\mathrm{Nb}$ shells on InAs nanowires. The nanowires are grown on $\mathrm{Si}(111)$ substrates by molecular beam epitaxy (MBE) without foreign catalysts in the vapor-solid mode. The metal shells are deposited by electron-beam evaporation in a metal MBE. High quality supercondonductor/semiconductor hybrid structures such as $\mathrm{Al} / \mathrm{InAs}$ and $\mathrm{Nb} / \mathrm{InAs}$ are of interest for ongoing research in the fields of gateable Josephson junctions and quantum information related research. Systematic investigations of the deposition parameters suitable for metal shell growth are conducted. In case of $\mathrm{Al}$, the substrate temperature, the growth rate and the shell thickness are considered. The substrate temperature as well as the angle of the impinging deposition flux are explored for Nb shells. The core-shell hybrid structures are characterized by electron microscopy and x-ray spectroscopy. Our results show that the substrate temperature is a crucial parameter in order to enable the deposition of smooth $\mathrm{Al}$ layers. Contrary, Nb films are less dependent on substrate temperature but strongly affected by the deposition angle. At a temperature of $200{ }^{\circ} \mathrm{C} \mathrm{Nb}$ reacts with InAs, dissolving the nanowire crystal. Our investigations result in smooth metal shells exhibiting an impurity and defect free, crystalline superconductor/InAs interface. Additionally, we find that the superconductor crystal structure is not affected by stacking faults present in the InAs nanowires.
\end{abstract}

\section{INTRODUCTION}

InAs nanowires are promising building blocks for two major present and future topics in research i.e. applications in quantum computing ${ }^{1,2}$ and the realization of Majorana fermions ${ }^{3,4}$. Combining application and fundamental research, these intersecting subjects can be tackled using superconductor/semiconductor (SC/SM) hybrid systems. For instance, the coupling of gate-defined qubits at the nanowire ends via a superconductor has been proposed ${ }^{2}$, but also quantum network structures ${ }^{5}$. Further, gateable nanowire-based Josephson junctions ${ }^{6-8}$ are in the focus of ongoing research. SC/InAs systems have been predicted to provide the optimal environment for proximity-induced p-wave superconductivity, leading in presence of an external magnetic field to spinless quasiparticles in form of triplet state Cooper pairs within the semiconductor ${ }^{4,9}$. Moreover, InAs is known to be easily proximitized if brought into an impurity-free contact with $\mathrm{Nb}$ or $\mathrm{Al}^{4,7}$. This means that a superconducting gap can be induced within the semiconductor. These two ingredients combined with a large g-factor ${ }^{10}$ and Rashba spin-orbit coupling ${ }^{11,12}$ are prerequisites for the appearance of Majorana fermions ${ }^{3}$. However, a clean SC/SM interface of high transparency is crucial to circumvent ambiguous experimental results and in order to provide an optimized performance for future devices ${ }^{13}$. Defects and impurities at the SC/InAs interface lead to parasitic subgap states which can be detrimental for topological quantum information ${ }^{14}$.

MBE grown SC/InAs hybrid nanowire structures exhibiting a distinct superconducting gap have been reported so far only by Krogstrup et al. ${ }^{13}$. They used $\mathrm{Al}$ as superconductor and phase pure InAs NWs grown by the $\mathrm{Au}$-catalyzed vapor-liquid-solid method on InAs (111)B substrates. In the mentioned report, the epitaxy of $\mathrm{Al}$ shells took place at a substrate temperature of about $30{ }^{\circ} \mathrm{C}$ and planar (on the substrate) $\mathrm{Al}$ growth rates of $0.3-0.5 \mathrm{umh}^{-1}$. Here, the Al-lattice orientations [111] and $[11 \overline{2}]$ have been observed ${ }^{13}$. In this communication we seek to extend the range of superconductors feasible for contacting to niobium. Furthermore, self-catalyzed InAs NW growth instead of Au-catalyzed growth is applied, paving the way for a Si compatible technology.

$\mathrm{Au}$-catalyzed growth can lead to the incorporation of $\mathrm{Au}$ impurities ${ }^{15-17}$ and the creation of deep level defects which deteriorate the electrical and optical properties and are thus not compatible with Si technology ${ }^{18}$. InAs NWs grown via the vapor-solid (VS) mode circumvent these obstacles, though they exhibit a strong polytypism. This manifests itself in a frequent switching between the zincblende (ZB) and the wurtzite (WZ) crystallographic phases, resulting in a high number of stacking faults.

In this paper, in-situ growth of the superconductor $\mathrm{Al}$ on VS grown InAs NWs using MBE is investigated. Our study extends the findings from metal deposition on phase pure wires ${ }^{13}$ to NWs with alternating ZB and WZ phases. We find that the crystal orientation of the deposited metals is not affected by the occurrence of stacking faults within the InAs, i.e. the polytypism. Further, it is observed that the temperature has a major influence on the morphology of the NW shell. This is expected as for low temperatures the diffusion length of the $\mathrm{Al}$ adatoms is diminished ${ }^{13}$. A degassing procedure 
was developed in order to evaporate the As film formed after InAs NW growth, preventing from the formation of an AlAs interlayer. Our studies result in an impurity free and crystalline $\mathrm{Al} / \mathrm{InAs}$ interface. Additionally, the deposition of niobium is analyzed highlighting the growth angle as the crucial parameter in order to obtain smooth and crystalline Nb shells. Nb possess the highest transition temperature and critical field of all elemental superconductors $^{19}$. Thus, the realization of $\mathrm{Nb} / \mathrm{InAs}$ hybrid structures is of particular interest.

\section{EXPERIMENTAL}

The growth of SC/InAs hybrids was realized in a state of the art cluster, interconnecting several ultra-highvacuum (UHV) chambers via transfer lines. This set up allows to conduct a multiple step growth process in situ. The InAs NWs have been grown in a III-V MBE chamber and the As capping which forms after growth has been degassed in a second As-free chamber. Subsequently, the $\mathrm{Al}$ and $\mathrm{Nb} \mathrm{SCs}$ have been deposited in a metal MBE by electron-beam evaporation.

The NWs were grown in the $[\overline{1} \overline{1} \overline{1}]$ direction on $\mathrm{Si}(111)$ substrates in absence of foreign catalysts using the VS method. For the growth, clean substrates were specifically prepared. First, the native silicon dioxide of the Si substrate was removed by $\mathrm{HF}$ wet etching and DIwater. This was followed by treating the cleaned Si for $45 \mathrm{~s}$ with hydrogen peroxide ${ }^{20}$. This leads to the creation of pinholes inside a thin silicon dioxide layer, which serve as nucleation centers. After preparation, the substrates were transferred immediately into the load-lock chamber of the UHV-cluster.

The NWs were grown at a substrate temperature of $475^{\circ} \mathrm{C}$ opening the In and As shutters for 1:20 h. Here, an In effusion-cell and an As cracker-cell were used. The Ingrowth rate was adjusted to $0.1 \mathrm{\mu mh}^{-1}$ and the $\mathrm{As}_{4}$ beam equivalent pressure was set to about $3.00 \times 10^{-5}$ Torr. The typical NW length was about $1.5 \mu \mathrm{m}$ and its width about $120 \mathrm{~nm}$. After growth, a thin As film forms during the cool down, covering the wire. Before the $\mathrm{Al}$ deposition, a degassing procedure in a separate chamber was used to evaporate the arsenic and create a clean NW surface. First, the substrate was heated to $400{ }^{\circ} \mathrm{C}$ keeping this value for 10 minutes, then it was heated to $450^{\circ} \mathrm{C}$ using a 10 minutes ramp up. Here, the temperature was kept constant for 5 minutes, followed by a consecutive cool down to $25^{\circ} \mathrm{C}$. The metals $\mathrm{Al}$ and $\mathrm{Nb}$ were deposited via electron-beam evaporators at a partial pressure of $\mathrm{p}_{\mathrm{Al}}=0.75 \times 10^{-5}$ Torr and $\mathrm{p}_{\mathrm{Nb}}=1.5 \times 10^{-9}$ Torr, respectively. The partial pressure was measured by a residual gas analyzer (RGA) and kept constant during growth via a feedback loop. The planar growth rates were $\mathrm{GR}_{\mathrm{Al}}=0.97 \mathrm{\mu mh}^{-1}$ and $\mathrm{GR}_{\mathrm{Nb}}=0.26 \mathrm{\mu mh}^{-1}$, respectively. The deposition time of the shells was $300 \mathrm{~s}$ for $\mathrm{Al}$ and $565 \mathrm{~s}$ for Nb, resulting in thicknesses of about $40 \mathrm{~nm}$ and $23 \mathrm{~nm}$, respectively. Sub- zero temperatures were obtained by turning off all heat sources and loading the sample in the MBE chamber $15 \mathrm{~h}$ prior to growth. The temperature converged to a value of about $-6{ }^{\circ} \mathrm{C}$ after this time period. Even lower temperatures of about $-28^{\circ} \mathrm{C}$ were reached by substituting the cooling water of the heater with cold vapor from liquid nitrogen.

The wire morphology as well as the crystal structure have been investigated using scanning electron microscopy (SEM) scanning transmission electron microscopy (STEM) as well as transmission electron microscopy $(\mathrm{TEM})^{21}$.

\section{RESULTS \& DISCUSSION}

\section{A. Aluminum shell}

Systematic studies of the growth parameters suitable to produce a smooth aluminum surface as well as an impurity and defect free $\mathrm{Al} / \mathrm{InAs}$ interface were conducted. We found that deposition at a relatively high substrate temperature of $42^{\circ} \mathrm{C}$ as depicted in Figure 1 a), leads to a rougher morphology of the SC shell compared to depositions at lower temperatures, shown in Figure $1 \mathrm{~b}), \mathrm{c}$ ). Growth at $\sim-6{ }^{\circ} \mathrm{C}$ results in a much smoother metal layer compared to the deposition at about $42{ }^{\circ} \mathrm{C}$. However, a lowering of the temperature from $\sim-6{ }^{\circ} \mathrm{C}$ to $\sim-28^{\circ} \mathrm{C}$ did not change the growth morphology significantly as depicted in Figure $1 \mathrm{c}$ ). One possible ex-

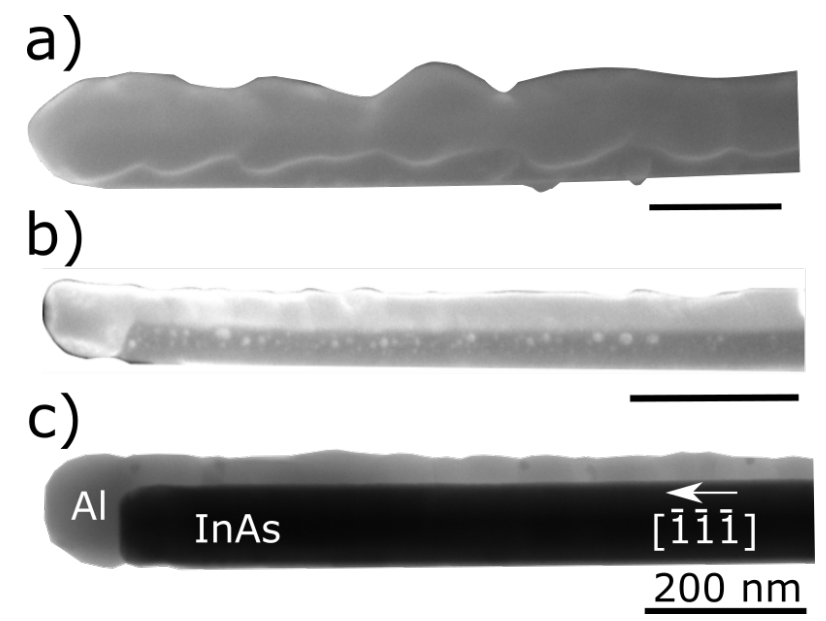

FIG. 1: Side views recorded with SEM in a), b) and STEM in c) depicting InAs NWs covered with an $\mathrm{Al}$ half shell of about $40 \mathrm{~nm}$ thickness, grown at different substrate temperatures: a) $\mathrm{T}_{\mathrm{s}}=42{ }^{\circ} \mathrm{C}$; b) $\mathrm{T}_{\mathrm{s}} \approx-6{ }^{\circ} \mathrm{C}$; c) $\mathrm{T}_{\mathrm{s}} \approx-28^{\circ} \mathrm{C}$.

planation for the temperature dependence of the shell morphology is given by the growth model developed by Krogstrup et al., reporting a similar trend (supplementary information of reference $\left.{ }^{13}\right)$. The main idea in this approach is that the diffusion length of the impinging 
$\mathrm{Al}$ adatoms decreases with decreasing substrate temperature $\mathrm{T}_{\mathrm{s}}$. This leads, for low $\mathrm{T}_{\mathrm{s}}$, to the formation of small islands which cluster while the out-of-plane orientation is dominated by surface energy minimization. The direction of lowest energy, here the [111] orientation, grows dominantly at an early growth stage, i.e. for thinner layers. At higher temperatures, the adatom diffusion length is larger, wherefore the islands exhibiting distinct lattice orientations grow bigger before they merge compared to the low temperature case. At a later growth stage, when the islands merge into clusters, the minimization of the grain boundary energy is predominant. Hence, the distinct grain orientation prevails, leading to a rougher metal shell morphology ${ }^{13}$.

Figure 2 a) shows an $\mathrm{Al} / \mathrm{InAs}$ interface in which the
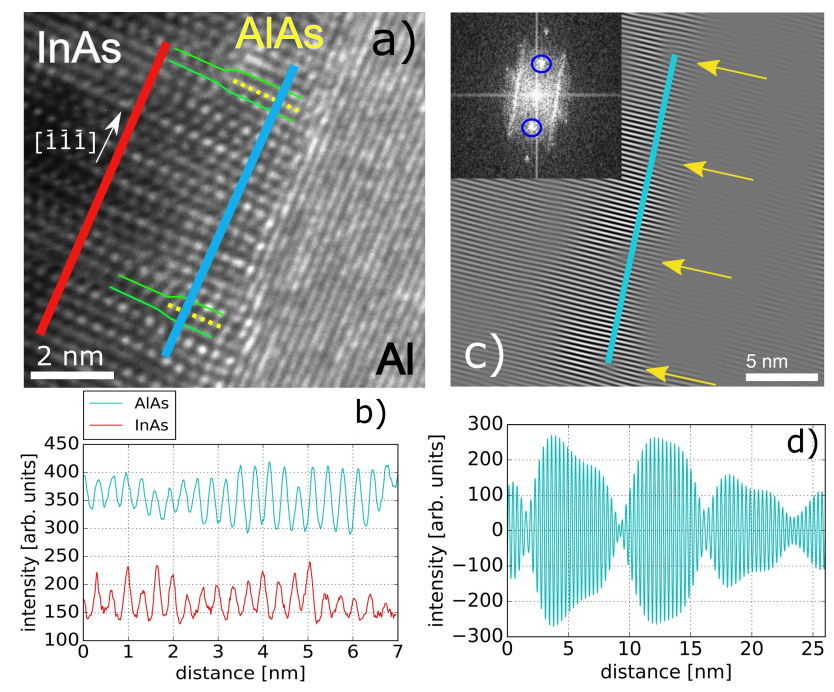

FIG. 2: a) TEM image of an InAs NW with an AlAs interlayer and $\mathrm{Al}$ shell. Intensity scans along the red and turquoise colored lines are plotted in b). The yellow dotted lines between the green lines highlight the edge dislocations in AlAs. b) Profiles according to the line

cuts of the AlAs and InAs to determine the lattice mismatch. c) Bragg filtered TEM image of an interface area to clearly indicate the distance between dislocations (marked with yellow arrows). The inset depicts the $\mathrm{ZB}[\overline{1} \overline{1} \overline{1}]$ reflex used for the Bragg filtered TEM image. The blue line cut showing the distance between three dislocations is plotted in $\mathrm{d}$ ).

InAs NW has not been treated with the degassing procedure introduced above prior to metal deposition. Thus, the InAs NW surface was covered with a thin layer of As. After Al deposition, an interlayer of a few nanometer thickness has formed, which we identified as AlAs. This assumption is based on estimations of the lattice mismatch between InAs and AlAs, explained below. The formation of an AlAs interlayer has been similarly observed by other groups ${ }^{13,22}$. The AlAs interlayer leads to the formation of defects close to the interface. As highlighted in Figure 2 a) by yellow dotted lines, mainly edge dislocations are observed. Defects are expected to be detrimental for Majorana bound states ${ }^{14}$ and might degrade the induction of a superconducting gap.

The lattice mismatch between the InAs wire and the AlAs interlayer has been estimated in two ways. The first estimation of the lattice mismatch is based on intensity line profiles as exemplarily shown in Figure $2 \mathrm{~b}$ ), from which the average lattice plane distances in the growth direction $\mathrm{d}_{\mathrm{InAs},[\overline{1} \overline{1} \overline{1}]}=0.339 \pm 0.007 \mathrm{~nm}$ and $\mathrm{d}_{\mathrm{AlAs},[\overline{1} \overline{1} \overline{1}]}=0.324 \pm 0.003 \mathrm{~nm}$ were extracted. The lattice mismatch is then given by $\mathrm{m}_{\text {InAs }}$ AlAs $=$ $\left(\mathrm{d}_{\text {InAs, }[\overline{1} \overline{1} \overline{1}]}-\mathrm{d}_{\mathrm{AlAs},[\overline{1} \overline{1} \overline{1}]}\right) / \mathrm{d}_{\mathrm{AlAs},[\overline{1} \overline{1} \overline{1}]}$ and was determined to $\mathrm{m}_{\text {InAs } / \mathrm{AlAs}} \approx 4.6 \%$. In the second method, Bragg

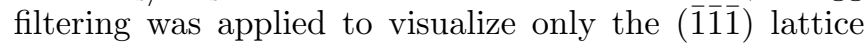
planes (Figure $2 \mathrm{c}$ )) from which the average dislocation distance $\mathrm{d}_{\text {Disloc. }}=7.197 \pm 0.002 \mathrm{~nm}$ was determined as indicated in Figure $2 \mathrm{c}$ ), d). The lattice mismatch is obtained via the relation $\mathrm{m}_{\mathrm{InAs} / \mathrm{AlAs}}^{\prime}=\mathrm{d}_{\text {InAs, }[\overline{1} \overline{1} \overline{1}]} / \mathrm{d}_{\text {Disloc. }}$ and was estimated to $\mathrm{m}_{\text {InAs/AlAs }}^{\prime} \approx 4.7 \%$. The extracted values are smaller than expected for bulk $(\sim 7 \%)$. This can be attributed to the occurrence of stacking faults which cause a diminished lattice plane spacing in comparison to phase pure wires. Moreover, the thin AlAs interlayer $(\delta \approx 2 \mathrm{~nm})$ is probably not completely relaxed, which leads to residual stress ${ }^{23}$, resulting in a lower mismatch.

Figure 3 depicts an $\mathrm{Al} / \mathrm{InAs}$ interface after metal depo-

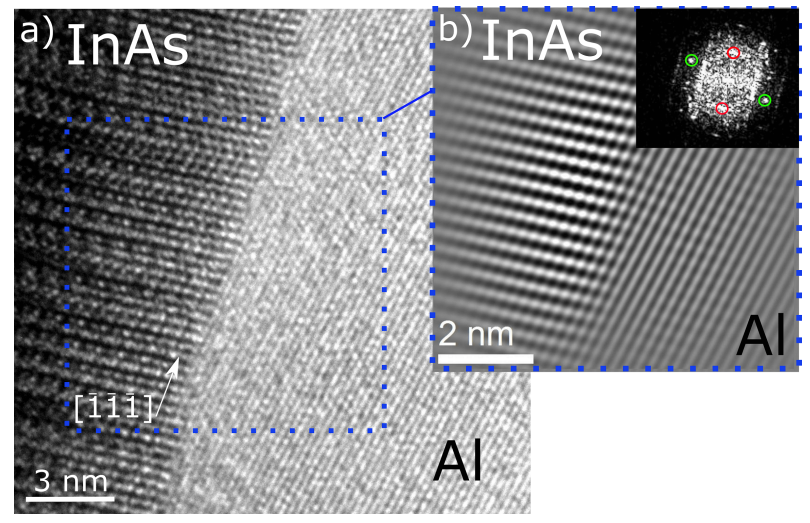

FIG. 3: a) TEM image of an InAs NW showing the InAs/Al interface after As evaporation. b) Bragg filtered TEM image (fast-Fourier transformation (FFT) followed by an inverse FFT (IFFT)) depicting a section (blue dotted box) of the TEM image presented in a) to spotlight the absence of dislocations. The inset shows the FFT of the TEM image depicted in a). Here the ZB [1̄11] reflexes of InAs are labeled red and the $\mathrm{Al}$ reflexes are labeled green, both are chosen for the IFFT.

sition with prior evaporation of the As layer formed after wire growth. An abrupt, impurity and defect free interface between the superconductor and the InAs nanowire is presented in Figure 3 a). The Bragg filtered image depicted in Figure $3 \mathrm{~b}$ ) exhibits, in contrast to Figure 2 b), no formation of dislocations, suggesting the absence 
of an AlAs interlayer. Thus, a defect and dislocation free $\mathrm{Al} / \mathrm{InAs}$ interface can be assumed. We notice that no In clusters have been observed due to the annealing procedure for As evaporation.

The WZ/ZB polytypism of the InAs NWs does not affect the Al lattice structure, i.e. the phase switching characteristic of the NWs does not influence the crystal orientation of the SC shell. It means that no epitaxial growth of $\mathrm{Al}$ on InAs is present for NWs showing polytypism. This finding is an extension to the epitaxial growth for phase pure wires presented in literature ${ }^{13}$. The Al shell grows polycrystalline, as illustrated in Figure 4 a)-c). However, long segments of several hundred nanometers with the same crystalline orientation are observed, as exhibited in Figure $4 \mathrm{a}$ ). The different lattice orientations are depicted in Figure $4 \mathrm{~d}$ )-f). The TEM images of the Al shell presented are superimposed by Al crystal simulations according to different $\mathrm{Al}$ directions in order to identify the lattice configuration. The simulations were created by using $\mathrm{VESTA}^{24}$. We identify the low energy [111] direction depicted in Figure $4 \mathrm{~d}$ ). Furthermore, the Al shell shown in Figure 4 e) can be assigned to the $[11 \overline{2}]$ direction, though its slightly deviating as indicated by the black arrow above the overlay. The lattice depicted in Figure $4 \mathrm{f}$ ) corresponds either to the [100] or the $[11 \overline{2}]$ direction. Though, the $[11 \overline{2}]$ direction would be rotated by $\pi$ around the plane normal in comparison to the overlay presented in Figure $4 \mathrm{e}$ ). None of the images exhibits the transfer of the characteristic polytypism of the NW into the superconductor, i.e. a high crystalline quality of the $\mathrm{Al}$ shell is observed independent of the characteristics of the InAs core.

\section{B. Niobium shell}

Niobium was deposited on vertically grown InAs NWs at a constant partial pressure of $\mathrm{p}_{\mathrm{Nb}}=1.5 \times 10^{-9}$ Torr. The substrate temperature $\mathrm{T}_{\mathrm{s}}$ was varied between $-6{ }^{\circ} \mathrm{C}$ and $200{ }^{\circ} \mathrm{C}$. Two characteristic regimes of the $\mathrm{Nb}$ depositions are identified, below $90^{\circ} \mathrm{C}$ and around $200^{\circ} \mathrm{C}$. First, the observations made at $90^{\circ} \mathrm{C}$ and below are presented.

The SEM images of Nb-covered InAs NWs presented in Figure 5 exhibit a streaked, columnar-shaped morphology almost independently of $\mathrm{T}_{\mathrm{s}}$ within the temperature range examined. This is contrary to the observations made for aluminum, for which $T_{\mathrm{s}}$ is the pivotal parameter. The behavior is possibly linked to the shorter diffusion length of $\mathrm{Nb}$ adatoms in comparison to $\mathrm{Al}$. If the $\mathrm{Nb}$ adatoms are highly immobile they form mostly tiny clusters in close vicinity to their initial sites. As the deposition takes place under an angle of about $30^{\circ}$ between the electron-beam cell and the wire, the area behind these clusters is shadowed. This in turn might lead to the streaked columnar-like growth observed. In combination with the results obtained from the $\mathrm{Al}$ deposition, i.e. smoother shells for lower
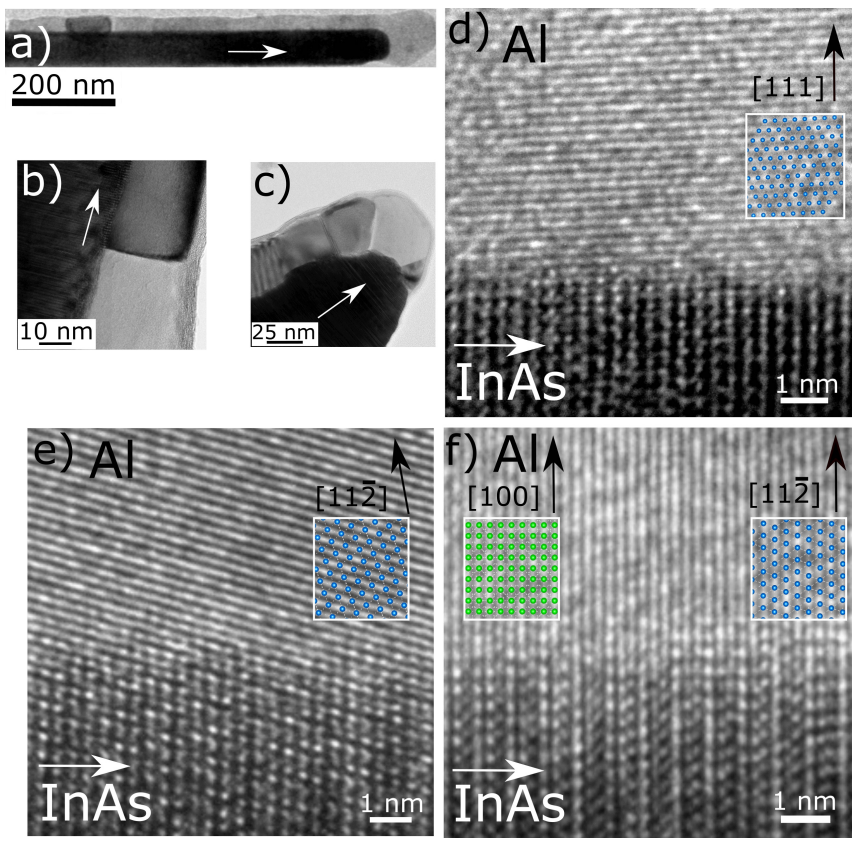

FIG. 4: a)-c) TEM images of $\mathrm{Al} / \mathrm{InAs}$ half shell structures. The metal deposition was conducted at $\mathrm{T}_{\mathrm{s}} \approx-6{ }^{\circ} \mathrm{C}$. d)-f) InAs and Al crystal structure with superimposed lattice simulations of $\mathrm{Al}$ (insets) to identify the crystal arrangement. d) Crystalline $\mathrm{Al}$ on InAs oriented in the [111] direction. e) Crystalline $\mathrm{Al}$ in $[11 \overline{2}]$ direction slightly deviating from wire normal as indicated by the black vector above the inset. f) $\mathrm{Al}$ in two possible orientations, indicated by the blue and green overlays. $\mathrm{Al}$ in $[11 \overline{2}]$ direction but rotated by $\pi$ around the plane normal compared to (e), indicated in

blue. Al in $\langle 100\rangle$ direction, indicated in green. The

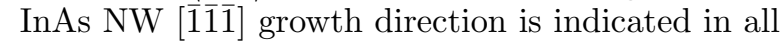
images by the white arrows.




C)

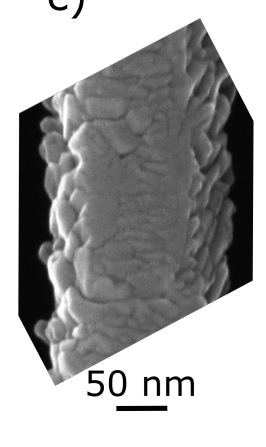



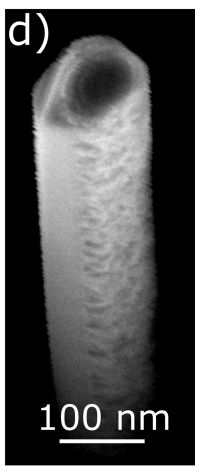

FIG. 5: Nb shells on InAs NWs at different substrate temperatures $\mathrm{T}_{\mathrm{s}}$. a), b), d) The shells depicted are about $23 \mathrm{~nm}$ thick; (c) The Nb shell is about $45 \mathrm{~nm}$ thick due to a longer deposition time.

temperatures, the findings indicate the existence of an 
optimal diffusion length for each material suitable to form smooth and crystalline metal shells at a certain deposition angle. This length can be controlled via the substrate temperature. Within the temperature range investigated $\left(\sim-6{ }^{\circ} \mathrm{C}\right.$ to $\left.90^{\circ} \mathrm{C}\right)$ shells on vertical grown NWs remained rough, independently of the layer thickness (c.f. Figure 5). Hence, in case of $\mathrm{Nb}$ apparently, a substrate temperature above $90^{\circ} \mathrm{C}$ is likely to allow for slightly smoother SC shells as the diffusion length is increased. Though, this temperature may already trigger chemical reactions between InAs and $\mathrm{Nb}$, as explained below, wherefore a different technique was applied.

Another approach enabling to overcome shadowing


FIG. 6: SEM pictures of silicon pyramids with and without InAs NWs. a) Side view of Si-pyramids with $\{111\}$ facets. b) Top view of InAs NWs on a Si pyramid.

due to $\mathrm{Nb}$ clusters forming at the initial growth stage, is to alter the deposition angle between the metal flux and the side facets of the wire. For this reason, $\operatorname{Si}(100)$ substrates were etched in $\mathrm{KOH}$ solution obtaining $\mathrm{Si}$ pyramids with $\{111\}$ facets $^{25}$ depicted in Figure 6 a). This enables the growth of InAs NWs on the Si $\{111\}$ facets of the pyramids as shown in Figure $6 \mathrm{~b}$ ), which allows the deposition of $\mathrm{Nb}$ shells under different angles.

The deposition geometry regarding the $\mathrm{Nb}$ flux and the side facets of the pyramids is sketched in Figure 7 a),b). The $\mathrm{Nb}$ shell morphology illustrated in Figure $7 \mathrm{c}), \mathrm{d})$ corresponds to the side facets $\mathrm{C}\left(\sim 87^{\circ}\right)$ and A $\left(\sim 25^{\circ}\right)$ of the pyramids sketched in Figure $\left.\left.7 \mathrm{a}\right), \mathrm{b}\right)$. Here, the substrate temperature is $40^{\circ} \mathrm{C}$. The shell morphology exhibits a strong dependence on the growth angle. Deposition under an angle of $87^{\circ}$ between the $\mathrm{Nb}$ flux and the wire leads to a smooth and closed surface, as illustrated in Figure $7 \mathrm{~d}$ ). This contrasts with depositions conducted at an angle of $25^{\circ}$ which are still streaked and columnar-shaped as depicted in Figure $7 \mathrm{c}$ ). The corresponding growth rates are estimated via SEM to $\mathrm{GR}_{25^{\circ}} \approx 0.3 \AA_{\mathrm{s}}^{-1}$ and $\mathrm{GR}_{87^{\circ}} \approx 0.7 \AA_{\mathrm{s}}^{-1}$, respectively. TEM images presented in Figure 8 confirm the different growth characteristics in dependence on the angle of deposition. Figure 8 a) depicts a smooth $\mathrm{Nb}$ shell on InAs of equal thickness, grown under an angle of about $87^{\circ}$. Here, apart from the InAs and the Nb, a $\mathrm{NbO}$ shell is visible. The $\mathrm{Nb}$ deposition conducted at an

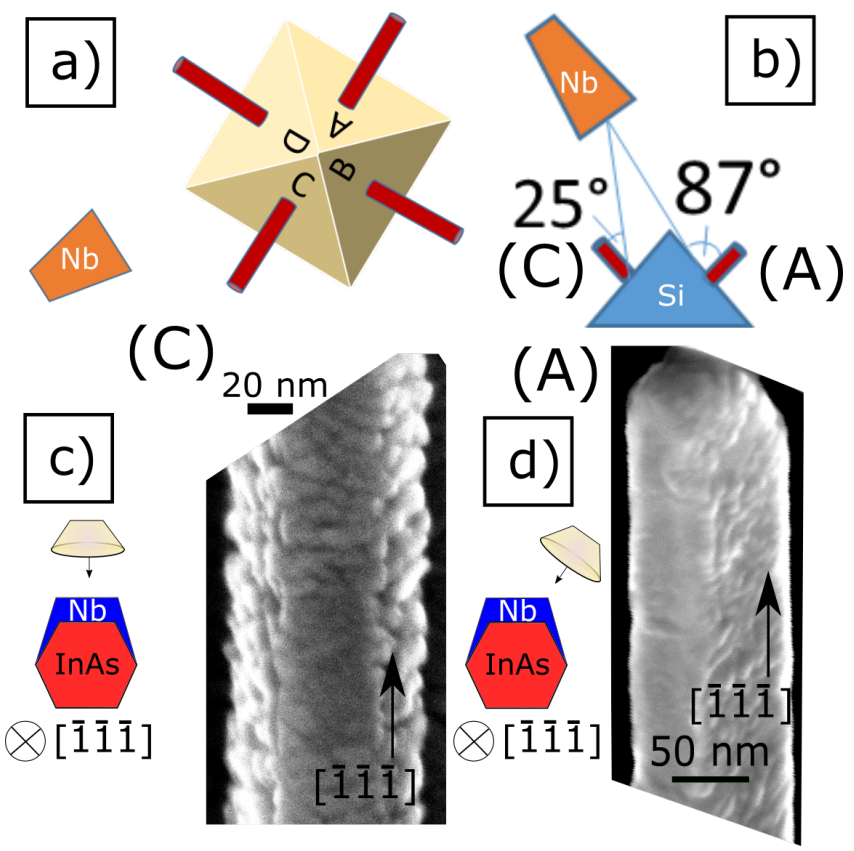

FIG. 7: a), b) Schematic illustrating the orientation of the $\mathrm{Nb}$ crucible in respect to NWs (red) on pyramid facets. Wires from the pyramid facet $\mathrm{C}$ and $\mathrm{A}$ are shown exemplary in c), d) labeled (C), (A), respectively. The viewing direction onto the $\mathrm{Nb}$ coated $\mathrm{InAs}$ wire is sketched at the left-hand side of the NW images in c) and $\mathrm{d})$.

angle of $25^{\circ}$ results in a columnar-shaped morphology as shown in Figure $8 \mathrm{c}$ ). The $\mathrm{Nb} / \mathrm{InAs}$ interface illustrated in Figure $8 \mathrm{~b}$ ) is impurity and defect free. The electron diffraction pattern shown in the inset of Figure 8 b) discloses the polycrystallinity of the $\mathrm{Nb}$ shell, as Debye-Scherrer rings occur. The streaks within the diffraction pattern are typical for InAs NWs exhibiting polytypism. Electrical investigations of $\mathrm{Nb}$ on InAs show that polycristalline growth does not strongly affect the superconducting properties ${ }^{26}$.

We observed that bending towards the coated side of the NWs occurs as shown in Figure $8 \mathrm{~d}$ ). Bending can be caused either by a difference in the thermal expansion coefficient or by a high lattice mismatch. The thermal expansion coefficients of InAs and $\mathrm{Nb}$ are $4.52 \mu^{\circ} \mathrm{C}^{-1}$ and $7.3 \mu^{\circ} \mathrm{C}^{-127}$, respectively. Hence, the $\mathrm{Nb}$ shell will contract stronger than the InAs, when cooled to ambient temperature after deposition, which could cause the observed bending towards the coated side. However, no opposite bending occurs if the covered wires grown on a planar substrate are heated up from $\sim-6^{\circ} \mathrm{C}$ to room temperature. No bending is seen, neither at $90^{\circ} \mathrm{C}$ nor at $40^{\circ} \mathrm{C}$ on vertically grown wires. In contrast, strong bending appears at $40^{\circ} \mathrm{C}$ on wires grown on pyramids, depicted in Figure $8 \mathrm{~d}$ ). These finding suggests that the wires are bend 

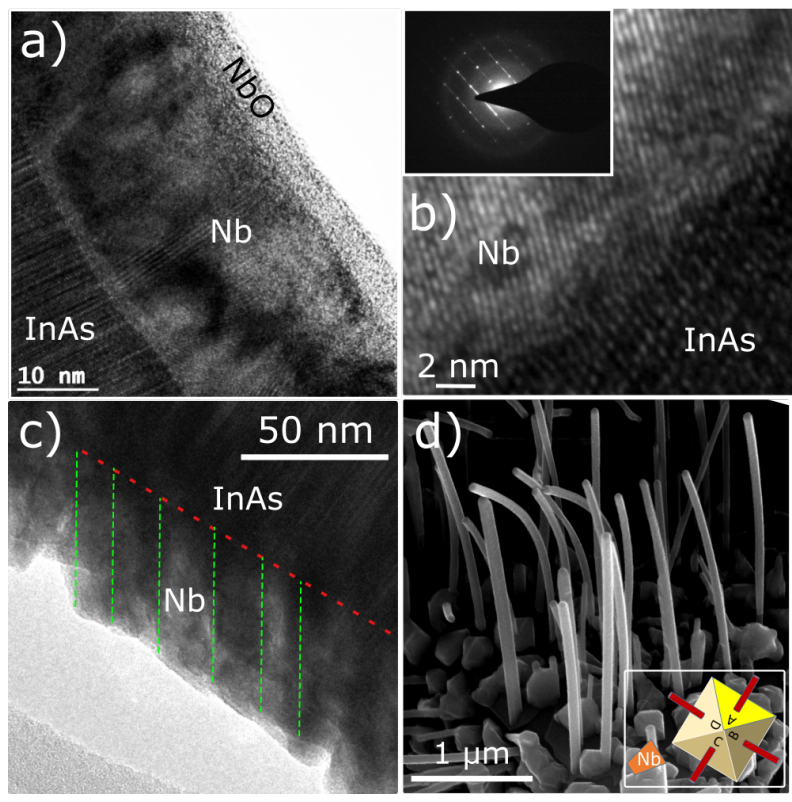

FIG. 8: a) TEM image showing a Nb shell on an InAs NW covered by a thin NbO layer. The deposition angle is $87^{\circ}$. The thin white film at the InAs interface is due to defocusing effects of the TEM. b) TEM image of the interface, exposing the crystal structure. The inset depicts the corresponding electron diffraction pattern. c) $\mathrm{Nb}$ shell deposited under $25^{\circ}$. The colored lines are guidance for the eyes, pointing out the columnar-shaped growth mode. d) SEM side view of Nb/InAs NWs grown on a (111) side facet (facet A) of a pyramid (labeled yellow in the inset indicating the deposition geometry).

TABLE I: Quantized atomic percentages extracted from the EDX scanned $\mathrm{Nb}$ shell deposited on an InAs NW at $200^{\circ} \mathrm{C}$ (c.f. Figure 9).

\begin{tabular}{l||l|l|l|} 
& As & In & Nb \\
\hline NW-Tip & $43 \%$ & $30 \%$ & $27 \%$ \\
NW-Body & $48 \%$ & $9 \%$ & $43 \%$
\end{tabular}

due to strain induced by a lattice mismatch and not due to thermal contraction. However, the strength of the bending depends on the NW diameter, wherefore thicker wires are less affected and only slightly bend.

Further, $\mathrm{Nb}$ depositions at a substrate temperature of $200^{\circ} \mathrm{C}$ were conducted. We observed that $\mathrm{Nb}$ reacts with InAs at this temperature. The NWs have partly dissolved at $200^{\circ} \mathrm{C}$, leaving empty $\mathrm{Nb}$ shells, as depicted in Figure 9 a1). The morphology of the Nb has changed, exhibiting a dotted surface. The EDX spectra depicted in Figure 9 a2), a3) show that In is still present in the NW tip, but diminished in the body of the wire. The different atomic percentages of the respective species, i.e. As, In and $\mathrm{Nb}$, between NW tip and body are listed in Table I). The high intensity $\mathrm{Cu}$ and $\mathrm{Al}$ peaks within the spectra stem from

the TEM grid and the sample holder, respectively but are not of importance for the analysis. EDX linescans (normal to the wire axis) of $\mathrm{Nb} / \mathrm{InAs}$ half shell structures for which $\mathrm{Nb}$ was deposited at $40{ }^{\circ} \mathrm{C}$ and $200^{\circ} \mathrm{C}$ are depicted in Figure 9 a4) and a5), respectively. Note that the shift of the peak positions is merely due to a flip of the wires. At a deposition temperature of $40^{\circ} \mathrm{C}$ the As and In signal strongly overlap and the $\mathrm{Nb}$ shell signal is clearly distinct. At $200^{\circ} \mathrm{C}$ in contrast, the $\mathrm{In}$ and As signal do not overlap anymore but a strong correlation between $\mathrm{Nb}$ and $\mathrm{As}$ is observed. This finding shows clearly that a chemical reaction between $\mathrm{Nb}$ and As was triggered at $200^{\circ} \mathrm{C}$ during the deposition. Figure 9 b1) shows the electron diffraction pattern of a $\mathrm{Nb}$ shell deposited at $200^{\circ} \mathrm{C}$. The occurrence of Debye-Scherrer rings is characteristic for polycrystalline materials. The absence of streaks demonstrates that the reaction with $\mathrm{Nb}$ at $200^{\circ} \mathrm{C}$ was detrimental for the InAs NW. Diffraction patterns of vapor-solid grown InAs NWs show characteristic streaks due to their high number of stacking faults, as exemplified in Figure 9 b2) for comparison ${ }^{29}$. The Debye-Scherrer diffraction pattern presented Figure 9 c) reveals that the $\mathrm{Nb}$ has reacted with As, forming new compounds. The obtained patterns show resemblance with the signatures of $\mathrm{NbAs}$ and $\mathrm{NbAs}_{2}$ but not with pure $\mathrm{Nb}$. We find that the substrate temperature sets a limit to the shell growth for two reasons. On the one hand, the deposition at $200^{\circ} \mathrm{C}$ is detrimental as the $\mathrm{Nb}$ reacts with the InAs, dissolving the InAs crystal. This was equally observed for depositions at a substrate temperature of $400{ }^{\circ} \mathrm{C}$. On the other hand, the $\mathrm{Nb}_{\mathrm{x}} \mathrm{As}_{\mathrm{y}}$ compounds formed are most likely not superconducting at ambient pressure anymore ${ }^{30,31}$ and therefore not suitable for $\mathrm{SC} / \mathrm{SM}$ hybrid structures. However, at $\mathrm{T}_{\mathrm{S}}=40^{\circ} \mathrm{C}$ no reaction between $\mathrm{Nb}$ and $\mathrm{InAs}$ is observed. The electron diffraction pattern presented in Figure 10 a) is clearly streaked, which proves the presence of an InAs NW. The side view of a niobium covered wire, presented in Figure $10 \mathrm{~b}$ ), discloses the homogeneous morphology of the $\mathrm{Nb}$ shell. The Debye-Scherrer diffraction pattern depicted in Figure $10 \mathrm{c}$ ), indicates no additional compounds to Nb, suggesting the absence of a chemical reaction at $40^{\circ} \mathrm{C}$. Hence, the structures produced constitute promising candidates for further research at low temperature. 


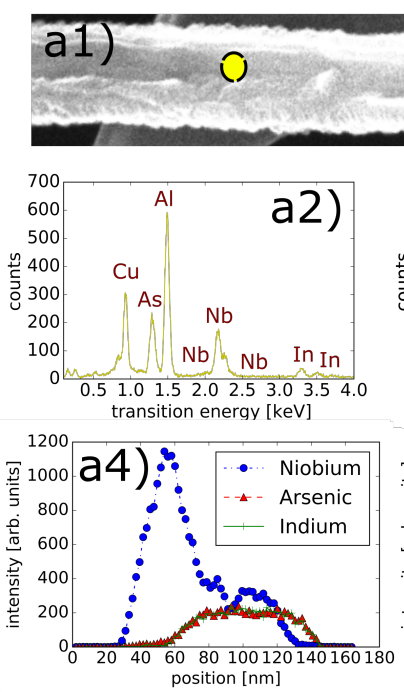

\section{$200 \mathrm{~nm}$}


FIG. 9: a1) Empty $\mathrm{Nb}$ shell stemming from $\mathrm{Nb}$ deposition on an InAs $\mathrm{NW}$ at $200{ }^{\circ} \mathrm{C}$ (scalebar on the right). a2), a3) EDX spectra recorded at the yellow dot (body) and blue dot (tip) in a1). The quantized atomic percentages of the tip spectrum shown in a2) as well as the body spectrum depicted in a3) are listed in Table I). a4, a5) EDX linescan (perpendicular to the wire axis) of a $\mathrm{Nb} / \mathrm{InAs}$ half shell structure where the shell was deposited at $40{ }^{\circ} \mathrm{C}$ (a4)) and $\left.200{ }^{\circ} \mathrm{C}(\mathrm{a} 5)\right)$. The legend for both plots is depicted in a4). b1)-b2) Electron diffractogram (inverted colors) from a $\mathrm{Nb}$ shell and an InAs NW, respectively. c) Debye-Scherrer ring radii extracted from b1). Nb compound patterns listed below for comparison ${ }^{28}$.
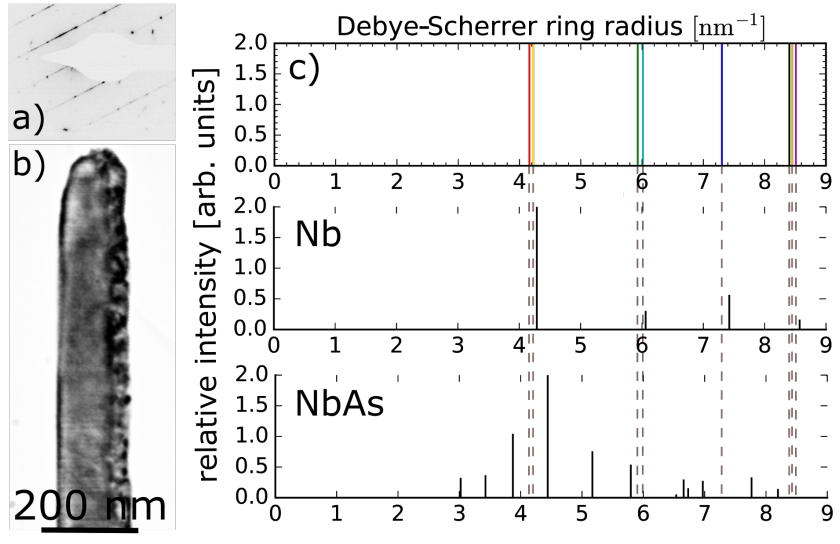

FIG. 10: a) Electron diffraction pattern (inverted colors) of the Nb half shell covered InAs NW depicted in b). b) TEM image of an Nb/InAs NW (wire on the left, shell on the right). The shell was deposited at $40^{\circ} \mathrm{C}$ under an elevation angle of $87^{\circ}$. c) Extracted Debye-Scherrer ring radii for a $\mathrm{Nb} / \mathrm{InAs} \mathrm{NW}$. Nb and NbAs Debye-Scherrer ring radii patterns are plotted for comparison $^{28}$.

\section{CONCLUSION}

In conclusion, we produced self-catalyzed $\mathrm{Al} / \mathrm{InAs}$ nanowire hybrid structures via MBE whereat the InAs NWs were grown within the vapor-solid mode. This report extends the investigations conducted on MBE grown SCs on phase pure InAs wires to SCs deposited on NWs exhibiting a high number of stacking faults. The hybrid structures disclose an impurity and defect free $\mathrm{Al} / \mathrm{InAs}$ interface with a crystalline $\mathrm{Al}$ shell. A degassing procedure was developed to evaporate the As film which forms after the NW growth. The wires possess a closed and smooth $\mathrm{Al}$ shell which is suitable for further processing. The SC is polycrystalline, although segments of several hundreds of nanometers show monocrystalline $\mathrm{Al}$ shell growth. We observed that the $\mathrm{Al}$ crystal is oriented within different directions which formation can be attributed to boundary and surface strain energy minimization ${ }^{13}$. Our findings show that the crystal structure of the $\mathrm{Al}$ shell is not affected by the polytypism present in the InAs core. No transfer of stacking faults at present within the InAs wire into the $\mathrm{SC}$ deposit is observed, suggesting that the $\mathrm{Al}$ crystal orientation is not predominantly defined by the NW crystal structure.

In order to extend the material combinations at hand, the growth of $\mathrm{Nb} / \mathrm{InAs}$ hybrids was investigated. We observed that the growth behavior is different from $\mathrm{Al}$, which can be attributed to the different diffusion length of the adatoms. In case of $\mathrm{Nb}$, the substrate temperature plays only a minor role. We found that the deposition angle, imposing a higher growth rate, changes the growth drastically. Smooth and coherent $\mathrm{Nb}$ shells can be produced by changing the angle between the $\mathrm{Nb}$ flux and the wire to $87^{\circ}$. The interface exhibits a crystalline structure and does not show any defects or impurities. 
The observation suggests a highly transparent interface suitable for low temperature investigations. Further, our findings revealed a chemical reaction between the InAs wire and $\mathrm{Nb}$ at a temperature of $200{ }^{\circ} \mathrm{C}$. The InAs crystal was dissolved, the arsenic reacting with the $\mathrm{Nb}$. As in the case of $\mathrm{Al}$, the $\mathrm{Nb}$ crystal structure seems not to be influenced by stacking sequence within the NW. This study is of importance to lay the groundwork for future quantum information technology devices based on superconducting hybrid structures. Crystalline SC shells and highly transparent SC/SM interfaces are crucial prerequisites within this field. As demonstrated, these requirements can be fulfilled using MBE growth. The realization of new material combinations such as $\mathrm{Nb} / \mathrm{I}-$ $\mathrm{nAs}$ in addition to $\mathrm{Al} / \mathrm{InAs}$ extends the temperature range and the critical fields possible for further research.

Acknowledgement. The authors thank Christoph Krause for the support at the MBE and the team of the Helmholtz Nano Facility for the assistance concerning the sample processing.
1 S. Nadj-Perge, S. M. Frolov, E. P. Bakkers, and L. P. Kouwenhoven, Nature 468, 1084 (2010).

2 M. Leijnse and K. Flensberg, Phys. Rev. Lett. 111, 060501 (2013).

3 A. Das, Y. Ronen, Y. Most, Y. Oreg, M. Heiblum, and H. Shtrikman, Nat. Phys. 8, 887 (2012).

${ }^{4}$ R. M. Lutchyn, J. D. Sau, and S. Das Sarma, Phys. Rev. Lett. 105, 077001 (2010).

5 J. Alicea, Y. Oreg, G. Refael, F. von Oppen, and M. P. A. Fisher, Nat. Phys. 7, 412 (2011).

6 S. Abay, D. Persson, H. Nilsson, F. Wu, H. Q. Xu, M. Fogelström, V. Shumeiko, and P. Delsing, Phys. Rev. B 89, 214508 (2014).

7 Y.-J. Doh, J. A. van Dam, A. L. Roest, E. P. A. M. Bakkers, L. P. Kouwenhoven, and S. De Franceschi, Science 309, 272 (2005).

8 S. Baba, J. Sailer, R. S. Deacon, A. Oiwa, K. Shibata, K. Hirakawa, and S. Tarucha, Appl. Phys. Lett. 107, 222602 (2015).

9 Y. Oreg, G. Refael, and F. von Oppen, Phys. Rev. Lett. 105, 177002 (2010).

10 M. T. Buörk, A. Fuhrer, A. E. Hansen, M. W. LarsSOn, L. E. Fröberg, and L. SAmuelson, Phys. Rev. B 72, 201307 (2005).

11 D. Liang and X. P. GaO, Nano Lett. 12, 3263 (2012).

12 S. Estévez Hernández, M. Akabori, K. SladeK, C. Volk, S. Alagha, H. Hardtdegen, M. G. Pala, N. Demarina, D. Grützmacher, and T. Schäpers, Phys. Rev. B 82, 235303 (2010).

13 P. Krogstrup, N. L. B. Ziino, W. Chang, S. M. AlBrecht, M. H. Madsen, E. Johnson, J. NygÅrd, C. M. Marcus, and T. S. Jespersen, Nat. Mater. 14, 400 (2015).

14 S. Takei, B. M. Fregoso, H.-Y. Hui, A. M. Lobos, and S. Das SARma, Phys. Rev. Lett. 110, 186803 (2013).

15 Y. A. Du, S. Sakong, and P. Kratzer, Phys. Rev. B 87, 075308 (2013).

16 J. E. Allen, E. R. Hemesath, D. E. Perea, J. L.
Lensch-Falk, Z. Y. Li, F. Yin, M. H. Gass, P. Wang, A. L. Bleloch, R. E. Palmer, and L. J. Lauhon, Nat. Nanotechnol. 3, 168 (2008).

17 M. Bar-Sadan, J. Barthel, H. Shtrikman, and L. Houben, Nano Lett. 12, 2352 (2012).

18 S. D. Brotherton and J. E. Lowther, Phys. Rev. Lett. 44, 606 (1980).

19 M. Peiniger and H. Piel, IEEE Trans. Nucl. Sci. 32, 3610 (1985).

20 T. Rieger, D. Grützmacher, and M. I. Lepsa, Phys. status solidi - Rapid Res. Lett. 7, 840 (2013).

21 M. Luysberg, M. Heggen, and K. Tillmann, J. largescale Res. Facil. JLSRF 2, A77 (2016).

22 N. L. B. Zimno, P. Krogstrup, M. H. Madsen, E. Johnson, J. B. Wagner, C. M. Marcus, J. NyGÅrd, and T. S. Jespersen, p. arXiv (2013).

23 A. Biermanns, T. Rieger, G. Bussone, U. Pietsch, D. Grützmacher, and M. Ion Lepsa, Appl. Phys. Lett. 102, 043109 (2013).

${ }^{24}$ K. Momma and F. Izumi, J. Appl. Crystallogr. 44, 1272 (2011).

25 T. Rieger, D. Rosenbach, D. Vakulov, S. Heedt, T. Schäpers, D. GrützmaCher, and M. I. Lepsa, Nano Lett. 16, 1933 (2016).

26 T. Akazaki, J. Nitta, and H. Takayanagi, Appl. Phys. Lett. 59, 2037 (1991).

27 Ioffe Institute, New Semiconductor Materials, http://www.ioffe.ru/SVA/NSM/Semicond/InAs/thermal.html, (accessed June 2016).

28 F.I.Z. KARLSRUhe, Inorganic Crystal Structure Database, https://icsd.fiz-karlsruhe.de, (accessed August 2016).

29 T. RIEGER, Growth and structural characterization of III$V$ semiconductor nanowires, Phd thesis, RWTH Aachen University, 2015.

30 J. Zhang, F. L. Liu, J. K. Dong, Y. Xu, N. N. Li, W. G. YANG, and S. Y. LI, p. arXiv:1506.00374 (2015).

31 B. Shen, X. Deng, G. Kotliar, and N. Ni, Phys. Rev. $B$ 93, 195119 (2016). 